INRA Prod. Anim., 2006, 19 (4), 279-286

\section{L'activité mitochondriale est un régulateur majeur de la différenciation des myoblastes et de l'expression des isoformes de myosine}

\author{
P. SEYER, S. GRANDEMANGE, L. PESSEMESSE, F. CASAS, G. CABELLO, C. WRUTNIAK-CABELLO \\ INRA, ENSAM, Université Montpellier 2, UMR 866, Différenciation Cellulaire et Croissance, \\ 2 place Pierre Viala, F-34060 Montpellier, France \\ Courriel : cabello@ensam.inra.fr
}

Bien que l'importance des mitochondries ait été longtemps restreinte à leur rôle dans la production d'ATP (Adénosine TriPhosphate) et à leur intervention dans le métabolisme énergétique, il est apparu plus récemment que ces organites sont impliqués dans les processus généraux du développement. Ainsi, la libération de facteurs de mort cellulaire par la mitochondrie est un événement clé qui conduit à l'apoptose (Newmeyer et Ferguson-Miller 2003). De plus, dans de nombreux types cellulaires, une inhibition de l'activité mitochondriale ralentit la prolifération (Leblond-Larouche et al 1979, Morais et al 1980, Van den Bogert et al 1992), de manière indépendante de la production d'ATP (Gregoire et al 1984, Buchet et Godinot 1998). Enfin, cette inhibition de l'activité de l'organite réprime également fortement la différenciation des érythroblastes (Kaneko et al 1988) ou des neurones (Vayssière et al 1992). Des résultats similaires ont été obtenus dans les myoblastes, quelle que soit l'espèce animale dont ils sont issus (Korohoda et al 1993, Herzberg et al 1993, Hamai et al 1997, Rochard et al 2000).

Les travaux de notre laboratoire ont établi que cette influence myogénique de l'activité mitochondriale résulte d'une véritable régulation, et n'est pas une simple conséquence d'une déficience du fonctionnement de l'organite, possible reflet des pathologies associées aux cytopathies mitochondriales. Pour cela, nous avons tiré parti de la découverte de la p43, récepteur mitochondrial de la triiodothyronine, qui est un facteur de transcription T3-dépendant spécifique du génome mitochondrial (Wrutniak et al 1995, Casas et al 1999). Sa surexpression dans les cellules en culture stimule en effet l'activité de la chaîne respiratoire et la mitochondriogenèse (Wrutniak et al 1995). L'utilisation de cet outil moléculaire, associée à celle d'inhibiteurs classiques de l'activité mitochondriale tels que le chloramphénicol, nous a permis de démontrer dans une même série d'expériences que si le chloramphénicol abroge la différenciation des myoblastes, cette dernière est fortement stimulée par la surexpression de p43 (Rochard et al 2000).

Ces études nous ont permis d'identifier plusieurs cibles impliquées dans l'influence myogénique de l'organite. D'une part, l'expression de Myogénine, un gène clé de l'induction de la différenciation terminale des myoblastes, est spécifiquement abrogée par le chloramphénicol et stimulée par surexpression de la p43 ; d'autre part, l'inhibition de l'activité mitochondriale abroge la capacité des facteurs myogéniques MyoD et Myogénine à induire la différenciation (Rochard et al 2000). Enfin, des résultats préliminaires de Differential Display suggéraient que deux autres gènes dont l'expression est modulée par l'activité mitochondriale pouvaient être impliqués dans cette régulation : c-Myc (Proto-oncogène) et Calcineurine.

L'objectif du travail de thèse présenté ici, avait pour but d'étudier l'implication de ces deux protéines dans l'influence myogénique de l'activité mitochondriale. Par ailleurs, compte tenu de l'implication de Calcineurine dans le déterminisme du phénotype contractile des fibres musculaires, nous avons étudié la possibilité selon laquelle l'activité mitochondriale pourrait réguler également l'expression des différentes isoformes de Myosines.

\section{1 / Implication de c-Myc} dans la régulation de la différenciation des myoblastes par l'activité mitochondriale

\section{1 / Le proto-oncogène c-Myc}

Le proto-oncogène c-Myc est un facteur de transcription ubiquitaire dont l'activité est dérégulée dans la plupart des processus oncogéniques. Il influence, directement ou indirectement, l'expression d'un ensemble de gènes impliqués dans la régulation de plusieurs processus cellulaires : contrôle du cycle cellulaire et de la prolifération, intervention dans les 
Figure 1. Principales fonctions de c-Myc dans la cellule. Parallèlement à son influence sur la synthèse protéique, le métabolisme cellulaire et la mitochondriogenèse, c-Myc intervient également dans la régulation de la prolifération et de la différenciation cellulaire ainsi que dans l'induction de l'apoptose, processus dérégulés au cours de la transformation cellulaire.

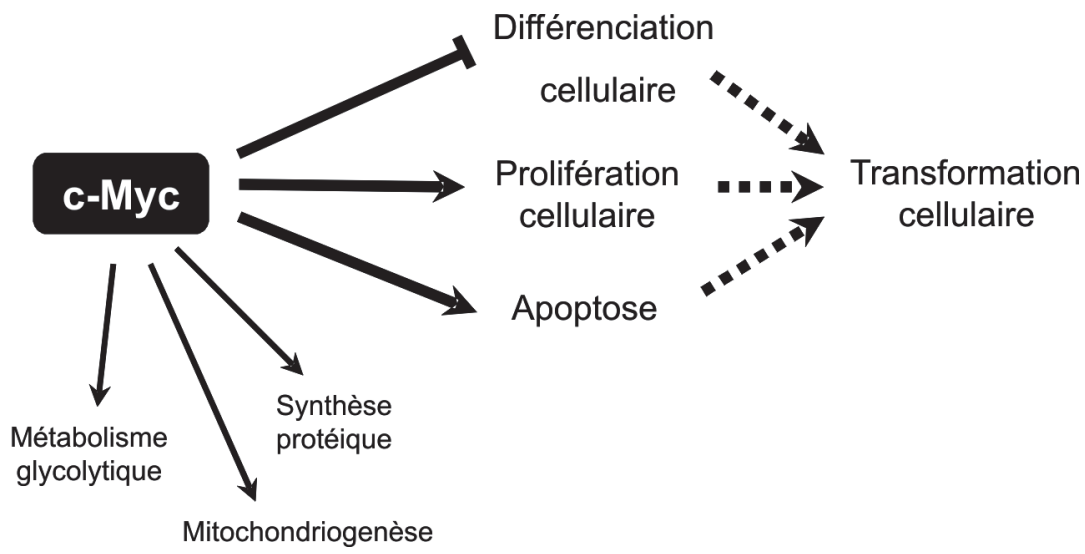

processus de différenciation ou d'apoptose (figure 1, Pelengaris et al 2002).

La majeure partie des fonctions biologiques de c-Myc requiert une interaction avec son partenaire majeur d'hétérodimérisation, Max. Ces dimères vont activer ou réprimer la transcription en se fixant sur des séquences spécifiques appelées boîtes E situées dans le promoteur de leurs gènes cibles (Claassen et Hann 1999). De plus, un grand nombre de partenaires d'interaction de c-Myc ou de Max module directement ou indirectement l'activité des dimères Myc-Max. Ces partenaires peuvent notamment interagir avec le domaine $\mathrm{N}$-terminal de c-Myc et réguler son interaction avec des complexes de la machinerie transcriptionnelle, ou interagir avec son domaine C-terminal ces protéines fait l'objet de régulations additionnelles. Enfin, la phosphorylation de la protéine c-Myc par différentes kinases module sa stabilité et son activité, et peut également influencer sa localisation cellulaire.

c-Myc apparaît ainsi comme un «master gene» situé au carrefour entre prolifération, différenciation et apoptose. Cette position stratégique qui détermine le devenir des cellules justifie cette régulation très fine de son expression et de son activité.

\section{2 / c-Myc est une cible de l'ac- tivité mitochondriale impliquée dans la régulation de la différen- ciation des myoblastes}

Des résultats préliminaires du laboratoire ont montré que l'inhibition de l'activité mitochondriale par le chloramphénicol stimule l'expression du proto-oncogène c-Myc dans les myoblastes aviaires. Or, la diminution de l'expression de c-Myc est considérée comme un événement nécessaire à l'induction de la différenciation, notamment dans les myoblastes murins et aviaires (Crescenzi et al 1994, La Rocca et al 1994). Ce facteur constituait donc un candidat particulièrement intéressant susceptible d'intervenir dans l'influence myogénique de l'organite.

a) L'expression et la localisation cellulaire de c-Myc sont régulées par l'activité mitochondriale

Dans les myoblastes aviaires, nous avons démontré que l'inhibition de l'activité mitochondriale par le chlo-

Figure 2. Différents niveaux de régulation de l'activité de c-Myc dans la cellule. L'activité de c-Myc au niveau cellulaire est étroitement contrôlée à tous les niveaux : expression (transcription du gène, stabilité de l'ARN (Acide Ribonucléique), traduction du messager, stabilité de la protéine), localisation cellulaire (translocation nucléaire), activité transcriptionnelle (partenaire de dimérisation et interactions avec des facteurs nucléaires).

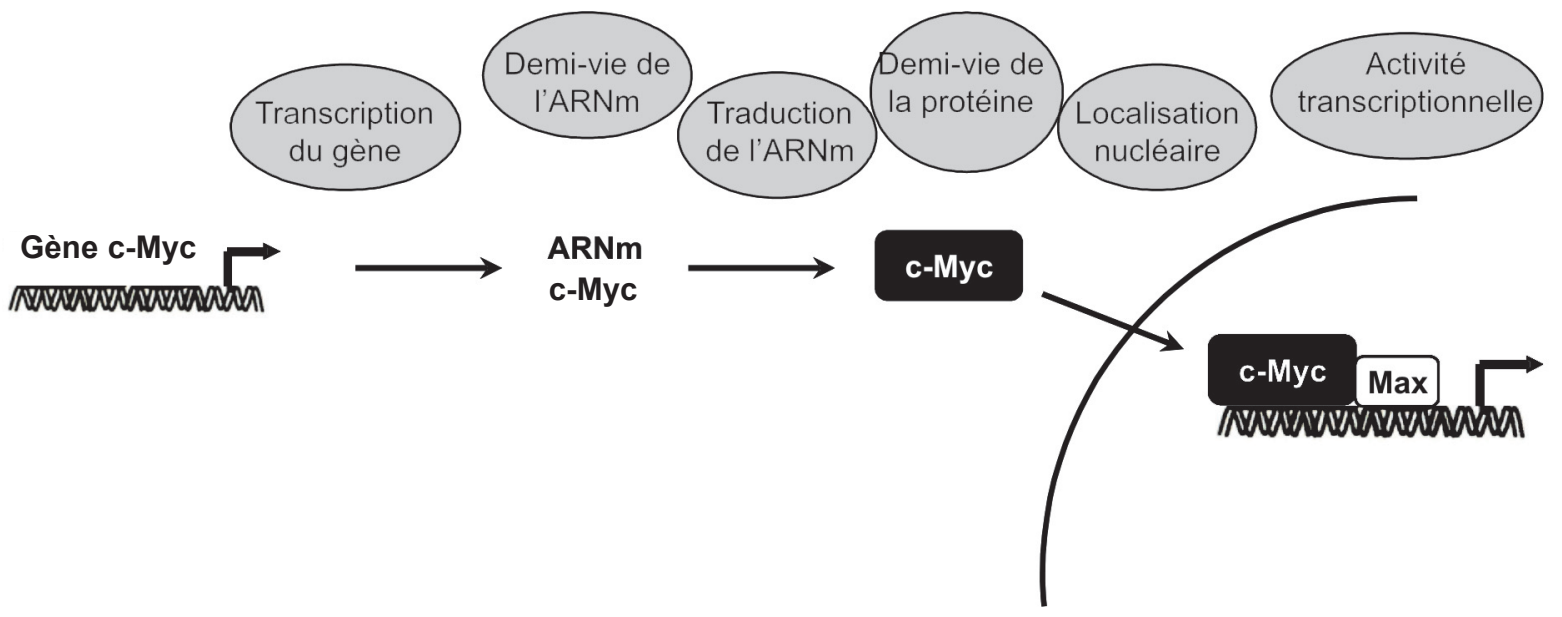


ramphénicol ne modifie pas l'expression de c-Myc dans les myoblastes prolifératifs, mais abroge la décroissance des niveaux des transcrits et de la protéine qui survient dès l'induction de la différenciation, par abaissement de la concentration sérique du milieu de culture à confluence cellulaire. Réciproquement, la stimulation de l'activité de l'organite par surexpression de p43 diminue l'expression de c-Myc à tous les stades de la culture, indépendamment de la concentration sérique du milieu. Cette observation concorde avec le fait que la surexpression de ce récepteur suffit à induire la différenciation des myoblastes même lorsqu'ils sont cultivés en milieu prolifératif riche en sérum (Rochard et al 2000). Ces résultats démontrent donc que l'expression de c-Myc est régulée par l'activité mitochondriale dès la transition prolifération/différenciation. Nous montrons également que cette régulation s'effectue en très grande partie via le contrôle de la stabilité du messager de c-Myc, qui est accrue par l'inhibition de l'activité mitochondriale, alors qu'elle est significativement réduite par la surexpression de p43.

Par ailleurs, contrairement à c-Myc murin, nous observons qu'une fraction importante du pool cellulaire de la protéine c-Myc aviaire est localisée dans le cytoplasme. Cette observation nous a permis d'établir que, dans cette espèce, il existe un niveau supplémentaire de régulation de l'activité de ce facteur de transcription par l'activité mitochondriale qui porte sur la localisation cellulaire de c-Myc. En effet, une inhibition de l'activité de l'organite par le chloramphénicol accroît la fraction de c-Myc localisée dans le noyau et donc son activité potentielle ; réciproquement, la surexpression de p43 semble diminuer la quantité de c-Myc nucléaire.

Cet ensemble de données valide donc l'hypothèse selon laquelle c-Myc constitue une cible de l'activité mitochondriale. En effet, non seulement l'expression de ce proto-oncogène est modifiée par l'activité mitochondriale via le contrôle de la demi-vie du transcrit, mais l'intensité de sa localisation nucléaire constitue un second niveau de régulation. Ainsi, toute augmentation de l'activité mitochondriale est associée à une inhibition de l'activité de c-Myc, via une diminution de son expression et une augmentation de sa rétention cytoplasmique, au moins dans les myoblastes aviaires. b) La surexpression de c-Myc reproduit les effets d'une inhibition de l'activité mitochondriale

La partie complémentaire de ce travail concernant $\mathrm{c}-\mathrm{Myc}$ a consisté à établir dans quelle mesure c-Myc, cible de l'activité mitochondriale, pouvait contribuer à l'influence myogénique de l'organite. En accord avec les résultats obtenus dans d'autres lignées de myoblastes (Denis et al 1987, Miner et Wold 1991, La Rocca et al 1994), la surexpression de c-Myc dans les myoblastes aviaires inhibe fortement leur différenciation terminale. De plus, alors que l'expression de MyoD n'est pas modifiée, celle de Myogénine décroît fortement, résultat à rapprocher de la régulation spécifique de l'expression de Myogénine par l'activité mitochondriale (Rochard et al 2000). Par ailleurs, comme une inhibition de l'activité mitochondriale par le chloramphénicol, la surexpression de c-Myc bloque totalement la capacité de Myogénine et CMD1 (MyoD aviaire) à induire la différenciation. Enfin, toujours comme un traitement au chloramphénicol, cette surexpression abroge la stimulation de la différenciation induite par p43. Or, l'expression de c-Myc est inhibée par surexpression de p43; le fait d'abolir l'influence myogénique de ce récepteur par restauration de l'expression de c-Myc par transfection indique que la diminution marquée de ce proto-oncogène constitue un élément clé de la stimulation de la différenciation par $\mathrm{p} 43$.

c) La sortie du cycle cellulaire est régulée par l'activité mitochondriale via le contrôle de l'expression de $c-M y c$

L'arrêt irréversible de la prolifération est une étape essentielle pour l'induction du programme de différenciation des myoblastes. Or, nous avons observé que l'influence du chloramphénicol sur la différenciation est négativement

Tableau 1. Comparaison entre l'influence d'une inhibition (chloramphénicol = chlor.) ou d'une stimulation (surexpression de p43) de l'activité mitochondriale et l'influence de la surexpression de c-Myc dans les myoblastes aviaires.

\begin{tabular}{|l|l|l|l|}
\hline & \multicolumn{1}{|c|}{ Chlor. } & c-myc & p43 \\
\hline Fusion des myoblastes & inhibition & inhibition & stimulation \\
\hline Différenciation biochimique & inhibition & inhibition & stimulation \\
\hline Expression de Myogénine & inhibition & inhibition & stimulation \\
\hline Expression de CMD1 & Pas d'effet & Pas d'effet & Pas d'effet \\
\hline $\begin{array}{l}\text { Activité myogénique de } \\
\text { Myogénine et CMD1 }\end{array}$ & inhibition & inhibition & N.D. \\
\hline Sortie du cycle cellulaire & inhibition & inhibition & stimulation \\
\hline
\end{tabular}

corrélée au nombre de myoblastes postmitotiques présents au moment de l'addition de la drogue. Cette observation suggérait donc que l'influence négative du chloramphénicol sur la différenciation s'exercerait essentiellement sur les myoblastes prolifératifs ; la sortie irréversible des myoblastes du cycle cellulaire (blocage dans la phase G0-G1) pourrait constituer une cible importante de l'activité mitochondriale. A l'appui de cette hypothèse, nous avons établi par analyse du cycle cellulaire que la fréquence des myoblastes en phase G0-G1 à confluence cellulaire est significativement augmentée par surexpression de la p43. Réciproquement, le chloramphénicol ou la surexpression de c-Myc diminue la proportion des myoblastes en phase G0-G1 au même stade de la culture. Il apparaît ainsi que, via le contrôle de l'expression et de l'activité de c-Myc, l'activité mitochondriale régulerait la sortie des myoblastes du cycle cellulaire, événement crucial de la différenciation des myoblastes. Il est à noter, concernant c-Myc, que ces résultats sont en accord sur d'autres types cellulaires, qui montraient que la surexpression de c-Myc bloque la sortie du cycle cellulaire (Resnitzky et Kimchi 1991, Goodrich et Lee 1992), alors qu'une inhibition de son expression induit la sortie du cycle et stimule la différenciation des lymphocytes T (Heikkila et al 1987).

d) Les mitochondries, c-Myc et la sortie du cycle cellulaire

En conclusion, nos résultats démontrent d'une part que l'expression de c-Myc est régulée négativement par l'activité mitochondriale, et d'autre part que la surexpression de c-Myc reproduit exactement l'influence d'une inhibition de l'activité mitochondriale par le chloramphénicol (tableau 1) : i) inhibition de la différenciation ; ii) inhibition de l'expression de myogéavec des données antérieures établies 
nine, sans influencer celle de MyoD ; iii) blocage de la capacité de MyoD et Myogénine à induire la différenciation; iv) abrogation de la capacité de la p43 à induire la différenciation ; v) inhibition de la sortie des myoblastes du cycle cellulaire. Cet ensemble de résultats démontre donc que le contrôle de l'expression de c-Myc constitue un mécanisme important par lequel les mitochondries régulent la transition prolifération/différenciation des myoblastes.

\section{2 / Implication de Calci- neurine dans la régulation de la différenciation des myoblastes et l'expression des isoformes de myosine par l'activité mitochon- driale}

\section{1 / Calcineurine, une phospha- tase de la signalisation calcique}

Calcineurine est une phosphatase impliquée dans de nombreux mécanismes physiologiques, notamment au niveau du développement. La protéine active se compose d'un trimère formé d'une sous-unité catalytique, CnA, sur laquelle se fixent la Calmoduline et la sous-unité régulatrice $\mathrm{CnB}$ (figure 3 ). En présence de calcium, la Calmoduline induit un changement de conformation de la sous-unité $\mathrm{CnA}$, libérant ainsi le site catalytique. La fixation de calcium sur la sousunité CnB jouerait un rôle structurel plutôt que régulateur (Shibasaki et al 2002).

L'activité phosphatase de la Calcineurine est régulée par la signali- sation calcique. Parmi les nombreux substrats de Calcineurine, les protéines NFAT (Nuclear Factor of Activated $T$ cells) constituent les cibles les mieux connues. Ce sont des facteurs de transcription, dont la déphosphorylation va induire la translocation vers le noyau. Par ailleurs, Calcineurine régule également l'activité transcriptionnelle des facteurs MEF2 (Liu et al 1997), une famille de facteurs de transcription notamment impliqués dans la régulation de la différenciation des myoblastes.

L'influence de Calcineurine a été initialement mise en évidence dans l'activation et la différenciation des lymphocytes $\mathrm{T}$ (Clipstone et Crabtree 1992, O'Keefe et al 1992). Depuis, elle a été impliquée dans une grande diversité de processus physiologiques, tels que la différenciation de plusieurs types cellulaires (Neal et Clipstone 2002, Tomita et al 2002, Hirotani et al 2004), la transformation des fibroblastes (Neal et Clipstone 2003) ou le contrôle du cycle cellulaire (Baksh et al 2000). En particulier, plusieurs travaux ont démontré l'implication de Calcineurine dans la différenciation musculaire, in vitro et in vivo (Olson et Williams 2000). De plus, cette phosphatase serait également un déterminant majeur du type contractile «lent» des fibres musculaires (Chin et al 1998, Bigard et al 2000, Serrano et al 2001, Naya et al 2000, Talmadge et al 2004).

Enfin, la capacité de Calcineurine à activer différentiellement des ensembles de gènes en fonction du type de signal calcique présent dans la cellule (amplitude, fréquence des pulses calciques) pourrait constituer un mécanisme de régulation de la plasticité musculaire, notamment au niveau de

Figure 3. Régulation de l'activité de Calcineurine par le calcium. L'activité de Calcineurine $A$ dépend de la fixation de la sous-unité Calcineurine $B$ et de la calmoduline en présence de calcium.

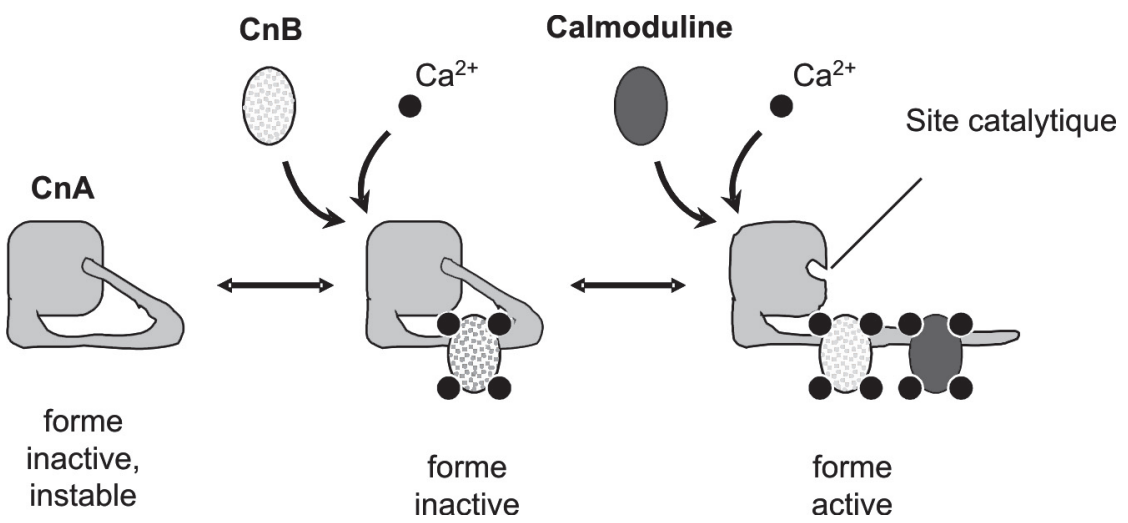

l'expression des diverses isoformes de myosine. Calcineurine pourrait ainsi constituer un effecteur de l'exercice ou de l'innervation impliqué dans les phénomènes de conversion du type contractile et métabolique des fibres musculaires.

\section{2 / Calcineurine est une cible de l'activité mitochondriale impliquée dans la régulation de la différenciation des myoblastes et l'expression des isoformes des chaînes lourdes de myosine}

Nos travaux antérieurs de Differential Display avaient permis d'identifier, dans les myoblastes aviaires, un fragment régulé par l'activité mitochondriale, présentant une forte homologie avec la séquence du messager de Calcineurine A humaine. Compte tenu de l'implication de Calcineurine à la fois dans la différenciation des myoblastes et dans la mise en place du phénotype contractile des fibres musculaires, la confirmation de la régulation de l'expression de cette phosphatase par l'activité mitochondriale constituait une piste particulièrement intéressante. En effet, un tel mécanisme pourrait permettre d'associer, sous une régulation commune, la mise en place des caractéristiques métaboliques et des caractéristiques contractiles des fibres musculaires.

a) L'expression de Calcineurine est régulée par l'activité mitochondriale

L'identification par RT-PCR utilisant des amorces correspondant au fragment précédemment identifié et à des séquences conservées du gène Calcineurine, nous a permis de confirmer que le fragment issu du Differential Display correspondait bien au transcrit de cette phosphatase calcium-dépendante. Ce travail a également permis d'établir la séquence de Calcineurine aviaire. Néanmoins, afin de tester son implication dans la différenciation des myoblastes et l'expression des diverses isoformes de myosine, nous nous sommes orientés sur un modèle de culture de myoblastes murins $(\mathrm{C} 2 \mathrm{C} 12)$, qui exprime les isoformes lentes et rapides de myosine, contrairement à notre lignée de myoblastes aviaires (QM7).

Nous avons tout d'abord démontré que, comme dans les myoblastes aviaires, l'inhibition ou la stimulation de l'activité mitochondriale respectivement abroge ou potentialise la différenciation des myoblastes $\mathrm{C}_{2} \mathrm{C}_{12}$. Ce résul- 
tat démontrait donc que l'influence myogénique de l'organite constitue un mécanisme général, qui n'est pas restreint aux seules espèces aviaires.

De plus, au niveau protéique, l'expression de Calcineurine est diminuée par une inhibition de l'activité mitochondriale par le chloramphénicol, alors qu'elle est accrue après surexpression de p43. Ces résultats confirmaient donc nos résultats antérieurs de Differential Display et démontraient que l'expression de cette phophatase est régulée par l'activité mitochondriale.

\section{b) Calcineurine est nécessaire à la différenciation des myoblastes}

Afin d'établir l'influence de la Calcineurine sur la différenciation des myoblastes, nous avons modifié 1'expression de cette phosphatase dans les myoblastes C2C12. L'utilisation d'un messager antisens qui diminue d'environ $40 \%$ l'expression de Calcineurine abroge totalement la différenciation des myoblastes et induit des modifications morphologiques importantes associées à des perturbations de l'organisation du cytosquelette ; de plus cette influence est associée à une extinction de l'expression de Myogénine. Réciproquement, l'expression d'une forme constitutivement active de Calcineurine stimule l'expression de Myogénine et la différenciation des myoblastes. Ces données suggèrent donc que i) après la sortie des myoblastes du cycle cellulaire, une expression minimale de Calcineurine est absolument nécessaire à la poursuite du programme de différenciation ; ii) Calcineurine est une cible de l'activité mitochondriale impliquée dans l'influence myogénique de l'organite, qui se situe, comme $\mathrm{c}-\mathrm{Myc}$, en amont de Myogénine.

c) Une stimulation de l'activité mitochondriale, ou la surexpression de Calcineurine, active l'expression des myosines lentes

La mise en évidence d'une régulation de l'expression de Calcineurine par l'activité mitochondriale nous a conduits à tester l'implication de l'organite dans l'expression différentielle des isoformes de myosine. Nous avons ainsi établi que la stimulation de l'activité mitochondriale induite par surexpression de p43 augmente très fortement l'expression des myosines lentes au détriment des myosines rapides ; réciproquement, l'inhibition de l'activité mitochondriale par le chloramphénicol qui bloque la différenciation sans abroger totalement l'expression des myosines, favorise l'expression des myosines rapides. De plus, en accord avec les données de la bibliographie, l'expression d'une forme constitutivement active de Calcineurine induit une augmentation significative de la proportion de MyHC (chaine lourde de la myosine) lente (Chin et al 1998, Delling et al 2000, Talmadge et al 2004).

Ainsi, ces résultats mettent pour la première fois en évidence une implication majeure des mitochondries dans la régulation de l'expression des isoformes de myosine, qui intervient dans la mise en place du type contractile des fibres musculaires. De plus, la régulation de l'expression de Calcineurine par l'activité de l'organite suggère fortement que cette phosphatase calcium-dépendante est un médiateur important dans cette régulation. Cependant, la surexpression de p43 augmente beaucoup plus fortement l'expression des myosines lentes que ne le fait l'expression d'une forme constitutivement active de Calcineurine. Ceci suggère donc que Calcineurine ne constitue probablement pas le seul facteur impliqué dans l'influence mitochondriale sur le type de myosine synthétisé dans les myoblastes.

d) L'activité mitochondriale, un déterminant majeur du développement des fibres de type lent oxydatif?

La démonstration que l'expression de Calcineurine est régulée par l'activité mitochondriale nous a permis d'identifier une nouvelle cible de l'organite, impliquée non seulement dans la régulation de la différenciation des myoblastes, mais également dans la régulation de l'expression de la MyHC lente. Cet ensemble de résultats suggère donc que l'activité mitochondriale influence la mise en place du type contractile des fibres musculaires. Cette implication dans l'orientation vers le phénotype lent, via Calcineurine, pourrait ainsi permettre d'expliquer l'association quasi permanente observée entre le type métabolique oxydatif et le type contractile lent dans les fibres musculaires (Lowry et al 1978, Delp et Duan 1996, Rivero et al 1999, Quiroz-Rothe et Rivero 2001).

\section{Conclusion : l'activité mi- tochondriale est un régula- teur majeur du développe- ment musculaire}

L'ensemble des résultats obtenus au cours de ce travail démontre que les mitochondries sont non seulement essentielles pour le fonctionnement du muscle squelettique via la production d'ATP, mais qu'elles sont également impliquées dans le développement de ce tissu. Nos travaux démontrent en effet que l'activité mitochondriale régule la différenciation des myoblastes via le contrôle de l'expression de c-Myc et de la sortie du cycle cellulaire, et via le contrôle de l'expression de Calcineurine, un effecteur positif de la différenciation. Nous montrons en particulier que ces deux voies sont impliquées dans le contrôle de l'expression de Myogénine, qui semble donc constituer une cible indirecte majeure de l'activité mitochondriale impliquée dans son influence myogénique (figure 4). De plus, la mise en évidence d'un contrôle de la différenciation par ces organites dans deux lignées différentes de myoblastes indique que cette régulation constitue probablement un mécanisme général, commun à plusieurs espèces animales.

La mise en évidence de la régulation de l'expression de ces gènes par l'activité mitochondriale est en accord avec de nombreux travaux démontrant l'existence d'un dialogue mitochondriesnoyau qui influence l'expression génique. Cependant, jusqu'alors, seuls des gènes impliqués dans la mitochondriogenèse avaient été identifiés. Ces résultats démontrent donc que l'importance de ce dialogue doit être étendu à la régulation de processus plus généraux tels que la différenciation cellulaire.

Enfin, nos résultats indiquent que, en partie via Calcineurine, l'activité mitochondriale est impliquée dans la régulation de l'expression des isoformes des chaînes lourdes de myosine, mécanisme susceptible d'expliquer l'association observée entre le type métabolique oxydatif et le type contractile lent des fibres musculaires.

Ces données obtenues in vitro restent à valider in vivo, travail actuellement en cours au laboratoire, via le phénotypage musculaire de souris invalidées pour ou surexprimant la $\mathrm{p} 43$. Les premiers résultats obtenus sont en accord avec les résultats présentés dans cet article. Enfin, la caractérisation de l'ensemble des gènes cibles de l'activité mitochondriale devrait apporter la connaissance d'autres gènes, régulés par l'organite, impliqués dans le développement musculaire ; ce travail en cours constitue la phase initiale d'un vaste projet destiné à identifier la nature de la signalisation mitochondriesnoyau à l'origine de l'influence myogénique des mitochondries. 
Figure 4. Schéma récapitulatif des mécanismes impliqués dans l'influence de l'activité mitochondriale sur la différenciation des myoblastes. La stimulation de l'activité mitochondriale, via une diminution de l'expression de c-Myc, stimule la sortie des myoblastes du cycle cellulaire et l'expression de Myogénine, induisant ainsi à une stimulation de la différenciation des myoblastes. De plus, en accroissant l'expression de Calcineurine, l'augmentation de l'activité de l'organite stimule non seulement la différenciation des myoblastes, mais favorise également l'orientation vers le type contractile «lent» des fibres musculaires. Ces données apportent une explication à l'association qui existe entre type métabolique oxydatif et type contractile lent des fibres musculaires.

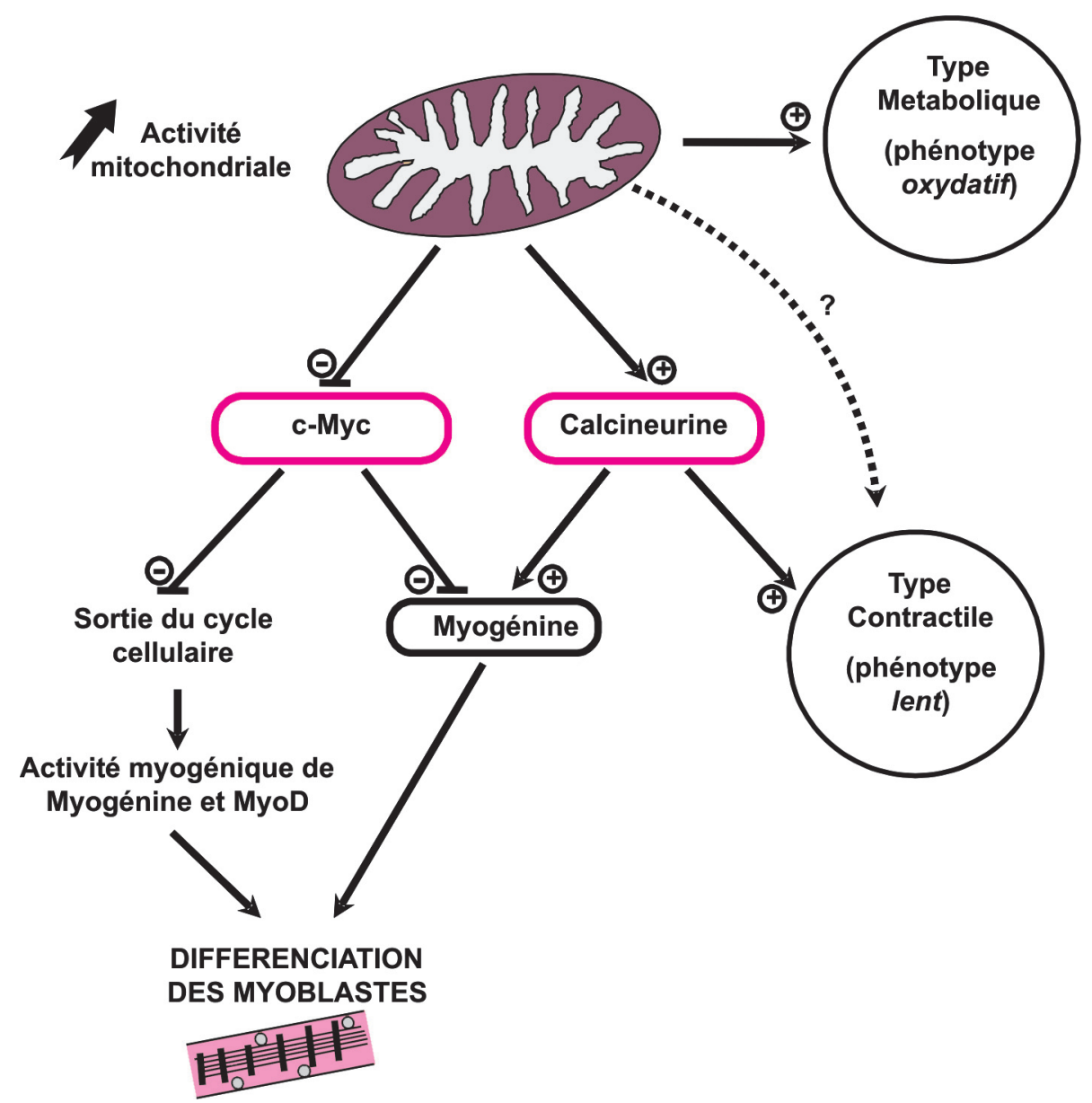

\section{Références}

Baksh S., DeCaprio J.A., Burakoff S.J., 2000. Calcineurin regulation of the mammalian $\mathrm{G} 0 / \mathrm{G} 1$ checkpoint element, cyclin dependent kinase 4. Oncogene, 19(24), 2820-2827.

Bigard X., Sanchez H., Zoll J., Mateo P., Rousseau V., Veksler V., Ventura-Clapier R., 2000. Calcineurin Co-regulates contractile and metabolic components of slow muscle phenotype. J. Biol. Chem., 275(26), 19653-19660.

Buchet K., Godinot C., 1998. Functional F1ATPase essential in maintaining growth and membrane potential of human mitochondrial DNA-depleted rho degrees cells. J. Biol. Chem., 273(36), 22983-22989.

Casas F., Rochard P., Rodier A., Cassar-Malek I., Marchal-Victorion S., Wiesner R.J., Cabello G., C. Wrutniak., 1999. A variant form of the nuclear triiodothyronine receptor c-Erb A alpha1 plays a direct role in regulation of mitochondrial RNA synthesis. Mol. Cell. Biol., 19(12), 79137924.

Chin E. R., Olson E.N., Richardson J.A., Yang Q., Humphries C., Shelton J.M., Wu H., Zhu W., Bassel-Duby R., Williams R.S., 1998. A calcineurin-dependent transcriptional pathway con- trols skeletal muscle fiber type. Genes Dev., 12(16), 2499-2509.

Claassen G. F., Hann S.R., 1999. Myc-mediated transformation: the repression connection. Oncogene, 18(19), 2925-2933.

Clipstone N. A., Crabtree G.R., 1992. Identification of calcineurin as a key signalling enzyme in T-lymphocyte activation. Nature, 357(6380), 695-697.

Crescenzi M., Crouch D.H., Tato F., 1994. Transformation by myc prevents fusion but not biochemical differentiation of $\mathrm{C}_{2} \mathrm{C}_{12}$ myoblasts: mechanisms of phenotypic correction in mixed culture with normal cells. J. Cell. Biol., 125(5), 1137-1145.

Delling U., Tureckova J., Lim H.W., De Windt L.J., Rotwein P. Molkentin J.D., 2000. A calcineurin-NFATc3-dependent pathway regulates skeletal muscle differentiation and slow myosin heavy-chain expression. Mol. Cell. Biol., 20(17), 6600-6611.

Delp M. D., Duan C., 1996. Composition and size of type I, IIA, IID/X, and IIB fibers and citrate synthase activity of rat muscle. J. Appl. Physiol., 80(1), 261-270.
Denis N., Blanc S., Leibovitch M.P., Nicolaiew N., Dautry F., Raymondjean M., Kruh J., Kitzis A., 1987. c-Myc oncogene expression inhibits the initiation of myogenic differentiation. Exp. Cell Res., 172(1), 212-217.

Goodrich D. W., Lee W.H., 1992. Abrogation by c-Myc of G1 phase arrest induced by RB protein but not by $\mathrm{p} 53$. Nature, 360(6400), 177-179.

Gregoire M., Morais R., Quilliam M.A., Gravel D., 1984. On auxotrophy for pyrimidines of respiration-deficient chick embryo cells. Eur. J. Biochem., 142(1), 49-55.

Hamai N., Nakamura M., Asano A., 1997. Inhibition of mitochondrial protein synthesis impaired $\mathrm{C}_{2} \mathrm{C}_{12}$ myoblast differentiation. Cell. Struct. Funct., 22, 421-431.

Heikkila R., Schwab G., Wickstrom E., Loke S.L., Pluznik D.H., Watt R., Neckers L.M., 1987. A c-Myc antisense oligodeoxynucleotide inhibits entry into $S$ phase but not progress from G0 to G1. Nature, 328(6129), 445-449.

Herzberg N. H., Zwart R., Wolterman R.A., Ruiter J.P., Wanders R.J., Bolhuis P.A., van den Bogert C., 1993. Differentiation and prolifera- 
tion of respiration-deficient human myoblasts. Biochim. Biophys. Acta, 1181, 63-67.

Hirotani H., Tuohy N.A., Woo J.T., Stern P.H., Clipstone N.A., 2004. The calcineurin/nuclear factor of activated $\mathrm{T}$ cells signaling pathway regulates osteoclastogenesis in RAW264.7 cells. J. Biol. Chem., 279(14), 13984-13992.

Kaneko T., Watanabe T., Oishi M., 1988. Effect of mitochondrial protein synthesis inhibitors on erythroid differentiation of mouse erythroleukemia (Friend) cells. Mol. Cell. Biol., 8, 3311-3315.

Korohoda W., Pietrzkowski Z., Reiss K., 1993. Chloramphenicol, an inhibitor of mitochondrial protein synthesis, inhibits myoblast fusion and myotube differentiation. Folia Histochem. Cytobiol., 31(1), 9-13.

La Rocca S. A., Crouch D.H., Gillespie D.A., 1994. c-Myc inhibits myogenic differentiation and myoD expression by a mechanism which can be dissociated from cell transformation. Oncogene, 9(12), 3499-3508.

Leblond-Larouche L., Morais R., Zollinger M., 1979. Studies of the effect of chloramphenicol, ethidium bromide and camptothecin on the reproduction of Rous sarcoma virus in infected chick embryo cells. J. Gen. Virol., 44(2), 323-331.

Liu S., Liu P., Borras A., Chatila T., Speck S.H., 1997. Cyclosporin A-sensitive induction of the Epstein-Barr virus lytic switch is mediated via a novel pathway involving a MEF2 family member. Embo J., 16(1), 143-153.

Lowry C. V., Kimmey J.S., Felder S., Chi M.M., Kaiser K.K., Passonneau P.N., Kirk K.A., Lowry O.H., 1978. Enzyme patterns in single human muscle fibers. J. Biol. Chem., 253(22), 8269-8277.

Miner J. H., Wold B.J., 1991. c-Myc inhibition of MyoD and myogenin-initiated myogenic differentiation. Mol. Cell. Biol., 11(5), 2842-2851.

Morais R., 1980. On the effect of inhibitors of mitochondrial macromolecular-synthesizing systems and respiration on the growth of cultured chick embryo cells. J. Cell Physiol., 103(3), 455466.
Naya F. J., Mercer B., Shelton J., Richardson J.A., Williams R.S., Olson E.N., 2000. Stimulation of slow skeletal muscle fiber gene expression by calcineurin in vivo. J. Biol. Chem., 275(7), 4545-4548.

Neal J. W., Clipstone N.A., 2002. Calcineurin mediates the calcium-dependent inhibition of adipocyte differentiation in 3T3-L1 cells. J. Biol. Chem., 277(51), 49776-49781.

Neal J. W., Clipstone N.A., 2003. A constitutively active NFATc1 mutant induces a transformed phenotype in 3T3-L1 fibroblasts. J. Biol. Chem., 278(19), 17246-17254.

Newmeyer D. D., Ferguson-Miller S., 2003. Mitochondria: releasing power for life and unleashing the machineries of death. Cell, 112(4), 481-490.

O'Keefe S. J., Tamura J., Kincaid R.L., Tocci M.J., O'Neill E.A., 1992. FK-506- and CsA-sensitive activation of the interleukin-2 promoter by calcineurin. Nature, 357(6380), 692-694.

Olson E. N., Williams R.S., 2000. Calcineurin signaling and muscle remodeling. Cell, 101(7), 689-692.

Pelengaris S., Khan M., Evan G., 2002. c-Myc: more than just a matter of life and death. Nat. Rev. Cancer, 2(10), 764-776.

Quiroz-Rothe E., Rivero J.L., 2001. Co-ordinated expression of contractile and non-contractile features of control equine muscle fibre types characterised by immunostaining of myosin heavy chains. Histochem. Cell Biol., 116(4), 299-312.

Resnitzky D, Kimchi A., 1991. Deregulated c-Myc expression abrogates the interferon- and interleukin 6-mediated G0/G1 cell cycle arrest but not other inhibitory responses in M1 myeloblastic cells. Cell Growth Differ., 2(1), 3341 .

Rivero J. L., Talmadge R.J., Edgerton V.R., 1999. Interrelationships of myofibrillar ATPase activity and metabolic properties of myosin heavy chain-based fibre types in rat skeletal muscle. Histochem. Cell Biol., 111(4), 277-287.
Rochard P., Rodier A., Casas F., Cassar-Malek I., Marchal-Victorion S., Daury L., Wrutniak C. Cabello G., 2000. Mitochondrial activity is involved in the regulation of myoblast differentiation through myogenin expression and activity of myogenic factors. J. Biol. Chem., 275(4), 2733-2744.

Sakamuro D., Prendergast G.C., 1999. New Myc-interacting proteins: a second Myc network emerges. Oncogene, 18(19), 2942-2954.

Serrano A. L., Murgia M., Pallafacchina G., Calabria E., Coniglio P., Lomo T., Schiaffino S. 2001. Calcineurin controls nerve activitydependent specification of slow skeletal muscle fibers but not muscle growth. Proc. Natl. Acad. Sci. USA, 98(23), 13108-13113.

Shibasaki F., Hallin U., Uchino H., 2002. Calcineurin as a multifunctional regulator. J. Biochem. (Tokyo), 131(1), 1-15.

Talmadge R. J., Otis J.S., Rittler M.R., Garcia N.D., Spencer S.R., Lees S.J., Naya F.J., 2004. Calcineurin activation influences muscle phenotype in a muscle-specific fashion. BMC Cell. Biol., 5(1), 28.

Tomita M., Reinhold M.I., Molkentin J.D., Naski M.C., 2002. Calcineurin and NFAT4 induce chondrogenesis. J. Biol. Chem., 277(44), 42214-42218.

Van den Bogert C., Spelbrink J.N., Dekker H.L., 1992. Relationship between culture conditions and the dependency on mitochondrial function of mammalian cell proliferation. J. Cell. Physiol., 152, 632-638.

Vayssiere J. L., Cordeau-Lossouarn L., Larcher J.C., Basseville M., Gros F., Croizat B., 1992. Participation of the mitochondrial genome in the differentiation of neuroblastoma cells. In Vitro Cell Dev. Biol., 28A(11-12), 763-772.

Wrutniak C., Cassar-Malek I., Marchal S. Rascle A., Heusser S., Keller J.M., Flechon J., Dauca M., Samarut J., Ghysdael J., Cabello G., 1995. A 43-kDa protein related to c-Erb A alpha 1 is located in the mitochondrial matrix of rat liver. J. Biol. Chem., 270, 16347-16354.

\section{Résumé}

Parallèlement à son rôle dans le métabolisme énergétique, l'activité mitochondriale intervient également dans l'induction de l'apoptose, ainsi que dans la régulation de la prolifération et de la différenciation cellulaires. Il existe en particulier une véritable régulation de la différenciation des myoblastes par l'activité mitochondriale, indépendante de la production d'ATP. Elle implique notamment le contrôle de l'expression de myogénine et de l'activité des facteurs myogéniques.

Dans cette étude, nous démontrons que l'expression du proto-oncogène c-Myc est respectivement stimulée ou diminuée par une inhibition ou une stimulation de l'activité mitochondriale. Cette régulation s'effectue en grande partie au niveau de la stabilité du messager, et au niveau de la localisation cellulaire de la protéine dans les myoblastes aviaires. De plus, la surexpression de c-Myc reproduit très exactement les effets d'une inhibition de l'activité mitochondriale : i) abrogation de la différenciation terminale ; ii) inhibition de l'expression de Myogénine, sans altération de celle de MyoD ; iii) blocage de l'aptitude des facteurs myogéniques à induire la différenciation ; iv) inhibition de la sortie des myoblastes du cycle cellulaire. Ces résultats démontrent que c-Myc est une cible importante de l'activité mitochondriale, impliquée dans l'influence de l'organite sur la différenciation des myoblastes.

Nous avons également mis en évidence l'existence d'un autre gène cible de l'organite qui code la phosphatase calcium dépendante Calcineurine. Son expression est respectivement inhibée ou stimulée par l'inhibition ou la stimulation de l'activité mitochondriale. De plus, l'expression d'une forme constitutivement active de Calcineurine stimule la différenciation des myoblastes et l'expression de Myogénine, alors que ces deux événements sont bloqués par l'expression d'un ARN antisens Calcineurine. Enfin, la stimulation de l'activité mitochondriale, comme l'expression d'une forme constitutivement active de Calcineurine stimule spécifiquement l'expression de l'isoforme lente des chaînes lourdes de myosine.

Ces données démontrent donc que, notamment via l'expression de Calcineurine, l'activité mitochondriale régule non seulement la différenciation des myoblastes, mais détermine également le type contractile des fibres musculaires. 


\begin{abstract}
Mitochondrial activity is a major regulator of myoblast differentiation and of the expression of myosin isoforms

Besides their influence on fuel metabolism, mitochondrial activity is also involved in the induction of apoptosis and in the regulation of cell proliferation and differentiation. In particular, an actual regulation of myoblast differentiation by mitochondrial activity is well established, independently of ATP synthesis, through the control of myogenin expression and myogenic factor activity.

In this study, we established that c-Myc expression is respectively up- or down-regulated by inhibition or stimulation of mitochondrial activity. This regulation essentially takes place at a post-transcriptional level and also concerns, in avian myoblast, the cellular localisation of the protein. In addition, c-Myc overexpression exactly mimics the influence of an inhibition of mitochondrial activity: i) inhibition of myoblast differentiation; ii) inhibition of myogenin expression; iii) inhibition of myogenic factor ability to induce differentiation; iv) inhibition of myoblast withdrawal from the cell cycle. These results demonstrate that c-Myc is an important target of mitochondrial activity involved in the myogenic influence of the organelle.
\end{abstract}

We also identified another target gene of mitochondrial activity encoding the calcium-dependent phosphatase calcineurin. Its expression is respectively inhibited or stimulated by inhibition or stimulation of mitochondrial activity. In addition, expression of a constitutively active form of calcineurin stimulates of myoblast differentiation and myosin isoform expression, whereas these two events are suppressed by expression of antisense calcineurin RNA. Lastly, stimulation of mitochondrial activity or overexpression of the constitutively active form of calcineurin specifically stimulates the expression of slow myosin heavy chain isoform.

These data demonstrate that, partly via calcineurin expression, mitochondrial activity not only regulates myoblast differentiation, but also the contractile type of muscle fibres.

SEYER P., GRANDEMANGE S., PESSEMESSE L., CASAS F., CABELLO G., WRUTNIAK-CABELLO C, 2006. L'activité mitochondriale est un régulateur majeur de la différenciation des myoblastes et de l'expression des isoformes de myosine. INRA Prod. Anim., 19, 279-286. 